cación y razones para abandonar el seguimiento) se tomaron de las historias clínicas. Los formularios llenados por los médicos se enviaron al centro coordinador en Monza para comprobar la coherencia de los datos. Las dudas surgidas se aclararon con los propios investigadores de manera individual. El seguimiento se extendió hasta el 30 de junio de 2000.

Se registraron 477 casos de abandono del seguimiento, $67 \%$ de ellos durante los tres primeros meses después del diagnóstico. Solo 40 casos $(8,4 \%)$ abandonaron el seguimiento al cabo de un año de haberse hecho el diagnóstico. El abandono del seguimiento puede considerarse un fracaso terapéutico, ya que lo más probable es que estos casos hayan muerto debido a la progresión de la enfermedad y a la falta de atención médica.

La supervivencia general a los tres años fue de $65,6 \%$ (error estándar $[\mathrm{ES}]=2,0$ ) cuando se excluyeron de los cálculos los casos de abandono del seguimiento $(16 \%)$, pero descendió a $54,2 \%$ (ES = $1,9)$ cuando los casos de abandono se sumaron a los fracasos terapéuticos.

El análisis detallado de los casos con leucemia linfoblástica aguda reveló una gran variabilidad entre los diferentes países que participaron en el estudio en cuanto a la supervivencia a los 3 años: $62,2 \%(\mathrm{ES}=5,3)$ en Cuba, $74,2 \%(\mathrm{ES}=3,3)$ en Costa Rica y $61,7 \%(E S=4,9)$ en Nicaragua, cifras todas muy superiores a las de los otros cuatro países participantes. Estos resultados pueden atribuirse a la baja proporción de casos que abandonaron el seguimiento en estos países $(0,9 \%, 2,7 \%$ y $7,5 \%$, respectivamente). En el caso de Cuba y Costa Rica, la alta retención de los casos durante el seguimiento podría deberse a la buena calidad de sus sistemas de salud, mientras que los pocos abandonos del seguimiento en Nicaragua posiblemente se debieron a los avances logrados recientemente por un programa nacional de hematología y oncología pediátrica. En países donde el número de casos que abandonaron el seguimiento en los primeros meses fue elevado se observaron las menores cifras de supervivencia a $\operatorname{los} 3$ años.

De estos resultados se pueden extraer dos conclusiones fundamentales: a) la curva general de supervivencia confirma y cuantifica la brecha que existe, en lo que respecta al derecho a la vida, entre los niños con cáncer que viven en países con menos recursos económicos y los que viven en países industrializados; b) las diferencias observadas en la supervivencia, incluso entre países de la misma región geográfica y cultural, hacen pensar que estos resultados no están tan vinculados con las condiciones macroeconómicas como con la existencia de una actitud de responsabilidad hacia la atención médica de los que la necesitan. Los casos de abandono temprano (durante el primer mes después del diagnóstico) constituyen la mayor proporción de casos con desenlaces desfavorables evitables.

Una larga tradición de atención continuada, respaldada por el concepto de que la salud es un derecho básico de las personas, genera perfiles de supervivencia similares en algunos países donde el tratamiento de los trastornos hematológicos y oncológicos, a pesar de su elevado costo, se considera esencial para la vida de una pequeña minoría compuesta de niños con cáncer. La promisoria situación de Nicaragua se debe en parte a la existencia de un proyecto de colaboración que garantiza la disponibilidad de medicamentos, pero más aun a la estrategia altamente participativa que se ha adoptado en ese país, en la que los padres toman parte en actividades de autoayuda que proveen las bases logísticas para poder cumplir con las diferentes etapas del tratamiento. (Valsecchi MG, Tognoni G, Bonilla M, Moreno N, Báez F, Pacheco C, et al. Clinical epidemiology of childhood cancer in Central America and Caribbean countries. Ann Oncol. 2004;15:680-5.)

\section{La relación del ambiente con la leptospirosis en Iquitos, Perú}

La leptospirosis es una zoonosis que se presenta de forma endémica o epidémica, tanto en áreas urbanas como rurales, en todas partes del mundo. La transmisión de Leptospira y la expresión clínica de la enfermedad varían en función del entorno ambiental y socioeconómico. La leptospirosis epidémica se acompaña de hemorragia pulmonar, insuficiencia renal e ictericia y es más frecuente en las áreas urbanas, donde el nivel de protección inmunitaria de los habitantes es más heterogéneo. Por el contrario, en las áreas rurales de los países en desarrollo se observa una mayor asociación entre la seropositividad a esta infección y la enfermedad subclínica.

El entorno ambiental de Iquitos, Perú, en la cuenca del Amazonas, presenta condiciones ideales para la transmisión de Leptospira, no solo debido al calor y a la humedad tropical predominantes, sino también a la elevada densidad poblacional y a la abundancia de mamíferos que pueden servir de vectores. Se ha observado que aproximadamente $30 \%$ de las personas de esta región que han padecido de fiebre aguda sin causa conocida presentan resultados serológicos que apuntan a la presencia de leptospirosis aguda.

El objetivo de este trabajo fue determinar la posible relación del ambiente con la exposición a Leptospira. Para ello se realizó un estudio seroepidemiológico transversal de base poblacional en tres contextos epidemiológicos diferentes del Perú: uno de alta prevalencia (Belén, un barrio marginal 
urbano de Iquitos), uno de prevalencia intermedia (Villa Buen Pastor, Moralillo y San Carlos, aldeas rurales de los suburbios de Iquitos) y un tercero de baja prevalencia (Las Pampas de San Juan de Miraflores, un suburbio pobre desértico de las afueras de Lima). También se investigó la presencia de Leptospira en ratas capturadas en el perímetro cercano a las viviendas, ya que son una fuente importante de transmisión de la infección.

Para documentar la exposición a la infección por Leptospira se empleó un ensayo combinado tipo ELISA que detecta inmunoglobulinas totales (IgM + IgG) en el suero. La detección de Leptospira en las ratas se realizó mediante la reacción en cadena de la polimerasa $(\mathrm{RCP})$ con ADN extraído de los riñones de los roedores.

En Belén participaron en el estudio 650 personas de 18 a 65 años de edad que vivían en 386 viviendas ( $18 \%$ de la población y $60 \%$ de las viviendas) entre febrero y octubre de 2001. La mediana del número de personas por vivienda fue de 5,8 (de 1 a 21). En las comunidades rurales participaron 316 personas de 65 años de edad o menos $(26 \%$ de la población local) entre febrero y agosto de 2000 y la mediana del número de personas por vivienda fue de 6,4 (de 1 a 24). En Las Pampas de San Juan de Miraflores se estudió a 150 personas $(0,4 \%$ de la población local) en abril de 1997; la mediana del número de personas por vivienda fue de 5,4 (de 1 a 14). Todos los participantes se seleccionaron al azar y parecían estar saludables.

La seroprevalencia en Belén fue de 28\% (182/ 650); no se observaron diferencias en las tasas de seropositividad entre los sexos o los grupos de edad, pero se encontró una tendencia ascendente del porcentaje de seropositividad a medida que aumentaba la edad $(P=0,018)$.

En las comunidades rurales de Iquitos, el porcentaje de seropositividad fue menor que en Belén $(16,5 \%$ frente a $28,0 \%$, respectivamente; $P<0,001)$ y no se encontraron diferencias según el sexo $(17,1 \%$ frente a $15,8 \% ; P=0,758)$. A pesar de que en los niños de 6 a 11 años de edad la prevalencia de anticuerpos contra Leptospira fue mayor que en los de 12 a 17 años, la seroprevalencia aumentó con la edad: de $4,0 \%$ en los niños menores de 5 años a $21,2 \%$ en las personas de 18 a 65 años $(P=0,012)$.

En los suburbios desérticos pobres (Las Pampas de San Juan de Miraflores) se encontró una seropositividad de $0,7 \%$ (1/150), la más baja de los tres contextos ambientales estudiados. En cuanto a la seroconversión, en Belén se detectaron 20 casos de infección $(12,4 \% ; 11$ mujeres y 9 hombres). La mediana del tiempo transcurrido entre la toma de las dos muestras fue de 157 días, que es el tiempo aproximado entre las temporadas de lluvia. La incidencia anual de seroconversión fue de 288 por 1000 personas. De las 34 personas que mostraron seropositividad durante el primer análisis serológico, 18 (53\%) dieron resultados negativos en el segundo, lo que puede indicar que los anticuerpos detectados - tanto del tipo IgM como del tipo IgG- tienen una vida muy corta.

Mediante el análisis unifactorial se encontró que los antecedentes de fiebre en los 6 meses anteriores al estudio serológico se asociaban con la seroconversión (razón de posibilidades $[R P]=3,29$; intervalo de confianza de 95\% [IC95\%]: 1,19 a 9,12; $P=0,022)$, asociación que se mantuvo significativa aun después de controlar los resultados según edad y sexo ( $R P=3,32$; IC95\%: 1,18 a 9,29; $P=0,023$ ). No se observaron casos graves de leptospirosis.

Se encontraron ratas infectadas con Leptospira en todas las localidades estudiadas. De 234 ratas atrapadas en Belén, 151 (64,0\%) eran de la especie Rattus rattus y 83 (36\%) eran $R$. norvegicus. De ellas, $25(10,6 \%)$ dieron resultados positivas a Leptospira en la RCP. La especie $R$. norvegicus presentó una mayor frecuencia de infección que $R$. rattus $(21,7 \%$ frente a $4,6 \%$, respectivamente; $P<0,001)$. La positividad en la RCP aumentó con la edad de la rata: $5,9 \%$ en las crías, $8,8 \%$ en las ratas juveniles y $12,0 \%$ en las ratas adultas, aunque esta tendencia no resultó estadísticamente significativa $(P=0,328)$. De las 41 ratas capturadas en San Juan de Miraflores (todas $R$. norvegicus), 2 (4,9\%) dieron resultados positivos en la RCP.

Este estudio presenta dos hallazgos importantes. En primer lugar, la exposición a Leptospira en lugares con condiciones ecológicas diferentes depende más del contexto ambiental que de la prevalencia de la infección en los roedores que circundan las viviendas. En segundo lugar, en Belén —área marginal urbana propensa a inundaciones, donde la exposición a la infección por Leptospira era alta-, la seroconversión estuvo asociada con la enfermedad sintomática (fiebre), pero no se notificaron casos graves.

Se encontraron dos factores de riesgo para la seropositividad a Leptospira: el nivel educacional y el vivir cerca de un río, particularmente en San José, un barrio de Belén. La prevalencia de anticuerpos contra Leptospira disminuyó en relación directa con el nivel educacional, posiblemente debido a una mejor higiene personal. Vivir en San José fue un factor de riesgo significativo en este estudio, tanto mediante el análisis unifactorial como con el multifactorial. Las ratas también presentaron una mayor tasa de infección en San José que en cualquier otra parte de Belén, lo cual indica que las tasas de transmisión de la leptospirosis pueden ser mucho mayores en localidades pequeñas específicas.

La alta frecuencia de exposición a Leptospira en Iquitos y su probable ubicuidad en este medio 
indican que la puesta en marcha de medidas de control para prevenir la leptospirosis en este entorno puede resultar difícil. La ausencia o gran escasez de casos graves de leptospirosis en esta región podría indicar que en esta población se han desarrollado mecanismos inmunitarios de protección. Se necesitan estudios prospectivos mayores de base poblacional para probar esta hipótesis. (Johnson MAS, Smith H, Joseph P, Gilman RH, Bautista $\mathrm{CT}$, Campos KJ, et al. Environmental exposure and leptospirosis, Peru. Emerg Infect Dis [serial on the Internet]. 2004;10(6). Hallado en: http:/ /www.cdc. gov/ncidod/EID/vol10no6/03-0660.htm. Acceso el 5 de junio de 2004.)

\section{Convocatoria para la presentación de manuscritos sobre la atención primaria de salud}

La Revista Panamericana de Salud Pública/Pan American Journal of Public Health solicita artículos de investigación originales y otros trabajos sobre la atención primaria de salud. Los materiales formarán parte de un número especial que se publicará en 2005 en conexión con las actividades conmemorativas que celebra la OPS en ocasión del vigésimo quinto aniversario de la primera Conferencia Internacional sobre Atención Primaria de Salud, que se celebró en Alma Ata, Kazastán (antigua Unión Soviética), del 6 al 12 de septiembre de 1978. La reunión dio como resultado la Declaración de Alma Ata, donde se estableció la meta de "salud para todos en el año 2000" y se delineó un plan de acción mundial centrado en la atención primaria de salud como elemento clave para alcanzar la meta planteada.

Los trabajos pueden redactarse en inglés, español o portugués y tratar cualquier tema que se relacione con la estructura, los procesos y los resultados propios de la atención primaria. Hay un interés particular en documentar los logros alcanzados en la Región desde la reunión de Alma Ata en lo que respecta a equidad, acceso, cobertura, sistemas de remisión de casos, y atención a las necesidades de grupos vulnerables; educación de salud y promoción de la salud; prevención, curación y rehabilitación; alimentación y nutrición; abastecimiento de agua potable y saneamiento básico; salud maternoinfantil y atención pediátrica y de la familia, incluida la planificación familiar; inmunización contra las principales enfermedades transmisibles; prevención y control de enfermedades endémicas; tratamiento adecuado de enfermedades y lesiones comunes; la provisión de medicamentos esenciales. Serán especialmente bienvenidos los estudios de evaluación de programas en América Latina y el Caribe que hayan dado resultados mensurablemente buenos o que constituyan ejemplos notables de participación comunitaria, acción intersectorial o uso eficiente de recursos financieros y humanos. También tendrán acogida los trabajos que describan el papel desempeñado por las organizaciones internacionales, agencias multilaterales, ONG y demás, así como los que examinen las áreas que aún requieren mayor desarrollo.

Todos los trabajos de investigación original serán sometidos a revisión por pares y su aceptación dependerá enteramente de su mérito científico. La fecha límite para la recepción de manuscritos en nuestra redacción es el 15 de septiembre de 2004.

\section{Call for Papers on Primary Health Care}

Revista Panamericana de Salud Pública/Pan American Journal of Public Health is seeking original research papers and other works dealing with primary health care. The papers are for a special issue that will be published in 2005 as part of PAHO's celebration of the twenty-fifth anniversary of the world's first International Conference on Primary Health Care, which was held in Alma Ata, Kazakhstan (former Soviet Union) on 6-12 September 1978. A result of that landmark event was the Declaration of Alma Ata, which established the goal of "health for all by the year 2000" and called for a worldwide plan of action that focused on primary health care as the key to attaining that goal.

Papers submitted for publication can be in English, Spanish, or Portuguese and can cover any issue related to structure, processes, and outcomes in primary health care. There is particular interest in documenting the strides made since Alma Ata in countries of the Region in terms of equity, access, coverage, referral systems, and attention to the needs of vulnerable segments of the population; health education and promotion; preventive, curative, and rehabilitative services; food supply and proper nutrition; supply of safe water and basic sanitation; maternal and child health care and family planning; immunization against the major infectious diseases; prevention and control of locally endemic diseases; appropriate treatment of common health problems and injuries; provision of essential drugs. Special consideration will be given to papers devoted to researching and evaluating primary health care programs in Latin America and the Caribbean that have yielded quantifiably good results or that are notable examples of community participation or inter-sectoral action or of efficient use of available financial and human resources. Materials on the role played by international organizations, multilateral agencies, NGOs, etc., will also be considered, along with opinion pieces on unfulfilled goals, ways to attain them, and areas that require improvement.

All original research papers will be submitted to peer review, and acceptance will depend entirely on scientific merit. The deadline for submission of all manuscripts is September 15, 2004.

\section{Dirección para el envío de manuscritos:} Address for submitting papers:

Publications

Pan American Health Organization

525 Twenty-third Street NW • Washington, DC 20037

Tel.: 202-974-3055 • Fax: 202 338-0869 